\title{
Hydrosomes: femtoliter containers for fluorescence spectroscopy studies
}

\author{
Ana M. Jofre, Jianyong Tang, Mark E. Greene, Geoffrey \\ M. Lowman, Nathan Hodas, et al.
}

Ana M. Jofre, Jianyong Tang, Mark E. Greene, Geoffrey M. Lowman, Nathan Hodas, Rani Kishore, Kristian Helmerson, Lori S. Goldner, "Hydrosomes: femtoliter containers for fluorescence spectroscopy studies," Proc. SPIE 6644, Optical Trapping and Optical Micromanipulation IV, 66440E (5 September 2007); doi: 10.1117/12.729515

SPIE Event: NanoScience + Engineering, 2007, San Diego, California, United States 


\title{
Hydrosomes: Femtoliter Containers for Fluorescence Spectroscopy Studies
}

\author{
Ana M. Jofre ${ }^{1}$, Jianyong Tang ${ }^{2}$, Mark E. Greene ${ }^{2}$, Geoffrey M. Lowman ${ }^{2}$, Nathan Hodas ${ }^{3}$, Rani \\ Kishore $^{1}$, Kristian Helmerson ${ }^{1}$, Lori S. Goldner ${ }^{2}$ \\ ${ }^{1}$ Atomic Physics Division, National Institute of Standards and Technology, Gaithersburg, MD \\ 20899 \\ ${ }^{2}$ Optical Technology Division, National Institute of Standards and Technology, Gaithersburg, MD \\ 20899 \\ ${ }^{3}$ Department of Physics, California Institute of Technology, Pasadena CA 91125
}

KEYWORDS: NANOCONTAINERS, NANODROPLETS, OPTICAL TRAPPING, SINGLE MOLECULE, ANISOTROPY, FLUORESCENCE SPECTROSCOPY, NANOCHEMISTRY, MICROREACTORS

\begin{abstract}
We report on improvements and innovations in the use of hydrosomes to encapsulate and study single molecules. Hydrosomes are optically-trappable aqueous nanodroplets. The droplets are suspended in a fluorocarbon medium that is immiscible with water and has an index of refraction lower than water, so hydrosomes are stable and optically trapped by a focused laser beam (optical tweezers). Using optical tweezers, we hold the hydrosomes within a confocal observation volume and interrogate the encapsulated molecule by fluorescence excitation. This method allows for long observation times of a molecule without the need for surface immobilization or liposome encapsulation. We have developed a new way for creating hydrosomes on demand by inertially launching them into the fluorocarbon matrix using a piezo-activated micropipette. Time-resolved fluorescence anisotropy studies are carried out to characterize the effects of the hydrosome interface boundary on biological molecules and to determine whether molecules encapsulated within hydrosomes diffuse freely throughout the available volume. We measured the fluorescence anisotropy decay of 20mer DNA duplexes, and enhanced green fluorescent protein (GFP). We conclude that the molecules rotate freely inside the nanodroplets and do not stick or aggregate at the boundary.
\end{abstract}

\section{HYDROSOMES: INTRODUCTION}

Hydrosomes are optically trappable femtoliter or sub-femtoliter $(\approx 700 \mathrm{~nm}$ diameter $)$ aqueous droplets in which we confine molecules for study by fluorescence spectroscopy. The droplets are suspended in a fluorocarbon medium, either 2-(nonafluorobutyl)heptafluorofuran (available from 3M as FC-77) or perfluorotriamylamine (available from 3M as FC-70). The solubility of water in FC-77 is $13 \mathrm{mg} / \mathrm{kg}$ and in FC-70 it is $8 \mathrm{mg} / \mathrm{kg}$, hence aqueous droplets formed in the medium are stable. The indices of refraction of FC-77 and of FC-70 are 1.28 and 1.30 respectively, while the index of refraction of water is 1.33 . This difference in refractive index enables optical trapping of the hydrosomes. We use optical tweezers at $1064 \mathrm{~nm}$ to hold the hydrosome within a confocal observation volume created by a visible probe beam, which fluorescently excites the molecule contained within the hydrosome.

Figure 1 shows a schematic of our experimental set up. The infrared optical tweezers are combined with the visible fluorescence excitation laser at a dichroic reflector and directed into a 1.4 numerical aperture objective. The emitted fluorescence is split between two detectors. For fluorescence resonance energy transfer (FRET) measurements, the emitted fluorescence is split into two bands with a second dichroic reflector. For polarization measurements, the fluorescence is split into two polarizations with a polarizing beam splitter. The inset of Figure 1 demonstrates single molecule detection within the hydrosomes.

Many single molecule measurements are done by recording the transit of single molecules through a confocal observation volume within a dilute solution. ${ }^{1}$ In this case, the fluctuations in the fluorescence intensity as a function of

Optical Trapping and Optical Micromanipulation IV, edited by Kishan Dholakia, Gabriel C. Spalding, Proc. of SPIE Vol. 6644, 66440E, (2007) · 0277-786X/07/\$18 - doi: 10.1117/12.729515 
time from molecules crossing the observation volume is analyzed using autocorrelation, giving information about the dynamics of the molecules on timescales shorter than the diffusion time across the observation volume (typically on the order of a millisecond). ${ }^{2}$ To capture slower conformational changes it is necessary to observe a molecule for longer periods of time. To this end, many single molecule experiments are done by immobilizing the molecule under study by binding $^{3,4,5}$ or adsorption ${ }^{6,7,8,9}$ onto a surface or in a gel. ${ }^{10,11}$ Another immobilization technique involves liposome encapsulation. ${ }^{12,13,14}$

The use of hydrosomes for single molecule spectroscopy has several advantages over surface attachment or liposome encapsulation for isolating and confining single molecules. Compared to surface attachment, sample preparation is considerably easier since no surface preparation or chemical tethering is necessary. Hydrosomes are also a more reproducible environment than are surfaces, avoiding the entropic and energetic interactions from the surface. Compared to liposome encapsulation, hydrosomes again provide much simpler sample preparation since no purification is necessary: all the analyte ends up in the hydrosome and none or negligibly little in the hydrophobic matrix. Finally, the use of hydrosomes facilitates mixing and the study of transiently interacting molecular complexes on an individual basis since hydrosomes fuse spontaneously. Liposome fusion requires membrane disturbance and risks losing contents to the aqueous matrix.

The use of hydrosomes for single molecule studies was first demonstrated by Reiner et al. ${ }^{15}$ They produced the hydrosomes, suspended in FC-77, using ultrasonic agitation. To facilitate droplet formation, trace quantities, $0.1 \%$ (v/v), of the surfactant Triton X-100 was added to the aqueous buffer. In their work, Reiner et al showed that approximately $50 \%$ of red fluorescent protein survived ultrasonication. We have found similar results with green fluorescent protein (GFP). However, we found that GFP does not survive ultrasonication without the use of a surfactant. The presence of surfactant either helps maintain the integrity of the protein or prevents it from partitioning out of the aqueous phase. We recently developed an alternative method for creating hydrosomes on demand that is useful in studies of single molecular complexes and that is less destructive to the hydrosome contents than ultrasonication.

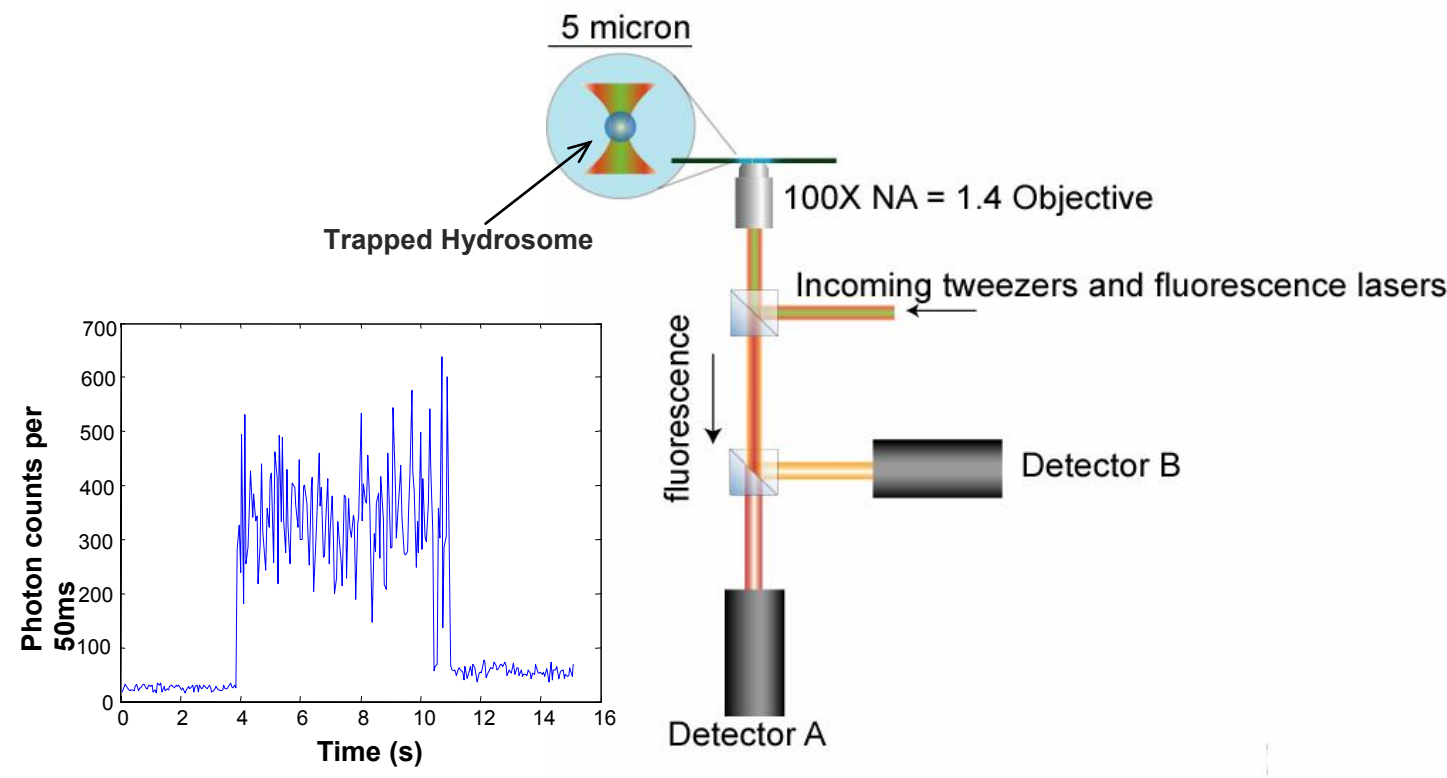

Figure 1: (right) Schematic diagram of the experimental apparatus used to trap and probe hydrosomes. An infrared laser at $1064 \mathrm{~nm}$ (IPG photonics YLD-5-1064) is used to optically trap the hydrosome. A visible laser is used to probe the molecules encapsulated within the hydrosomes. Fluorescence emission is detected by avalanche photodiode detectors (APDs). (left, inset) A fluorescence emission trace from a single Sulforhodamine B (SRB) molecule encapsulated within a hydrosome. A $532 \mathrm{~nm}$ visible laser (CrystaLaser GCL-532-L) was used to excite the SRB molecule (Sigma-Aldrich). The APD used to collect the photon counts shown above was a Perkin Elmer SPCM-AQR-14. 


\section{DIRECT DELIVERY OF HYDROSOMES ON DEMAND}

We have developed a technique for delivering hydrosomes one at a time on demand. The hydrosome injector is shown in Figure 2. It consists of a piezoelectric tube (EBL Products Inc. type EBL 2), a micropipette tip with hydrophobic coating, and backing pressure control from a precise pump (FemtoJet 5247, Eppendorf North America Inc.). The body of the injector is MACOR mounted onto an aluminum arm that is fixed on a three-axis translation stage. MACOR is also used to hold the tip and insulate it from the piezo. The entire assembly is mounted on or adjacent to the microscope and the micropipette tip is immersed into fluorocarbon and manipulated into the focal plane of the microscope. The piezo tube is wired to the output of a voltage amplifier ( $200 \mathrm{~V}$ max output), and a function generator synthesizes the sawtooth that drives the amplifier. A fast change of driving voltage leads to the quick retraction of the hydrophobic tip, and single small droplet is injected into the fluorocarbon. We are aware of two other devices that function in a fashion similar to this one. ${ }^{16,17}$

FC-70, which is nearly 20 times more viscous than FC-77, was used for forming and trapping injected hydrosomes. The high viscosity of FC-70 $(0.024 \mathrm{~Pa} \bullet \mathrm{s}))$ is necessary to facilitate droplet formation, and it also imparts a strong drag force on the ejected hydrosomes to slow them down for capture by the optical trap. We added $0.1 \%$ (v/v) of Triton x100 to our aqueous buffer to further facilitate droplet formation.

In this application, a backing pressure of about $300 \mathrm{hPa}$ created a meniscus at the tip. One cycle of a $200 \mathrm{~V}$ sawtooth applied to the piezoelectric tube then ejected a droplet from the tip into the matrix. The ejection force depends on the speed the tip can be retracted, which is limited by the bandwidth of the amplifier (1 MHz) and the mechanical resonances of the device. The droplets were then immobilized in a nearby optical trap.

The radius of a hydrosome was determined by injecting individual hydrosomes filled with dye at a known high concentration into a larger droplet that initially contained a very low known concentration of dye and whose size was easily measured (typically near 60 micron diameter). The small hydrosomes fuse immediately with the larger droplet and perturbed its volume by much less than one part in about 10,000, so to a good approximation the large droplet volume can be taken to be constant during the measurement. The fluorescence intensity from a point inside the large droplet (defined by the point spread function of the confocal system) is measured at fixed excitation power as a function of fused droplet number. By knowing initial dye concentrations and large droplet volume, we can infer the volume of the hydrosome from the slope of the intensity versus droplets fused curve. The deviations from a straight line put an upper limit on the spread in the droplet size. For one typical micropipette we find hydrosomes with diameter $736 \mathrm{~nm} \pm$ $31 \mathrm{~nm}$ or volume $212 \mathrm{aL} \pm 27 \mathrm{aL}$. These measurements and others for determining droplet size will be discussed in more detail in an upcoming publication.

Figure 2 shows both a schematic diagram of the device, and a picture of the micropipette ejecting droplets into flowing FC-70. The figure also shows that we are able to detect single molecules in hydrosomes formed with the injector technique. 

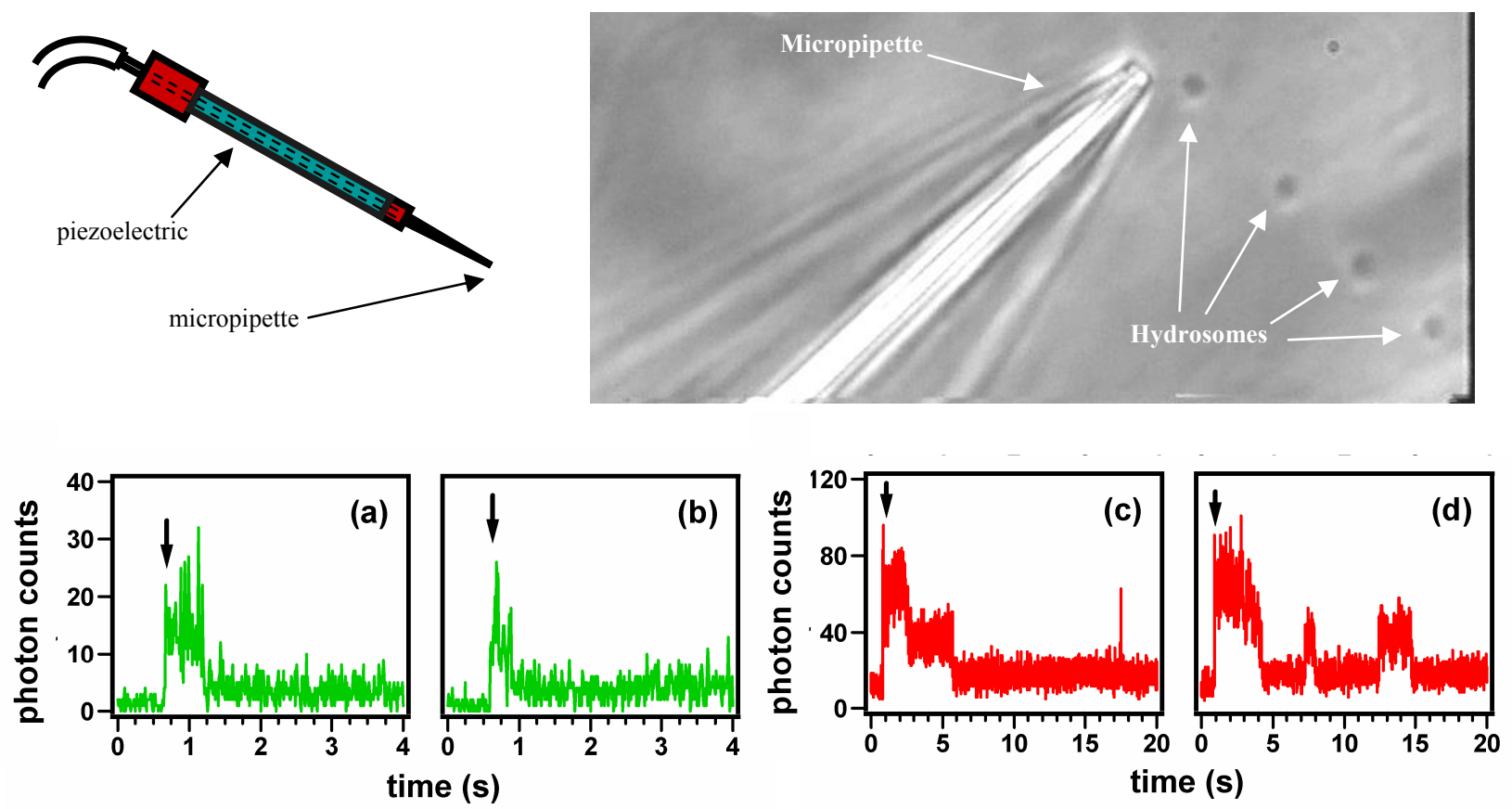

Figure 2: (Top left) A schematic diagram of our hydrosome injector. A micropipette tip is mounted onto a piezoelectric tube, and a backing pressure of about $300 \mathrm{hPa}$ is applied, forming a meniscus at the end of the tip. A sawtooth voltage applied to the piezo ejects a droplet from the micropipette. (Top right) A demonstration of the piezo-activated micropipette ejecting hydrosomes into an FC-70 medium, one per sawtooth cycle. The outer diameter of the micropipette tip is approximately one micron. (Bottom row) Single molecule bleaching events of $(a, b)$ EGFP and (c, d) Sulforhodamine B (SRB) isolated in hydrosomes that were formed using the hydrosome injector. Arrows indicate when the excitation laser is switched on. Counts were collected in $10 \mathrm{~ms}$ bins. A $532 \mathrm{~nm}$ laser was used to excite the SRB. A $488 \mathrm{~nm}$ laser was used to excite the EGFP.

\section{ROTATIONAL DYNAMICS INSIDE HYDROSOMES}

To probe the dynamics or conformational changes of individual molecules confined inside a hydrosome, and to use hydrosomes as reaction vessels, it is crucial to ensure that the molecules are freely diffusing and not aggregating at or sticking to the water-oil interface. We therefore measured the rotational motion of two biomolecules, a protein and a nucleic acid, inside hydrosomes using time-resolved fluorescence anisotropy (anisotropy decay) measurements. For comparison, we also made time-resolved fluorescence anisotropy measurements of the molecules in free solution.

We measured the anisotropy decay of enhanced green fluorescent protein (EGFP, from BioVision) suspended in phosphate buffered saline and of 20mer DNA duplexes tagged with Alexa 488 (from IDT) suspended in Tris buffer. The Alexa 488 fluorophore was attached via an NHS-Ester modification onto a 6-Carbon tether amino modification at the 5' end of one of the 20mer DNA oligos. All of the anisotropy measurements were done at $3 \mu \mathrm{mol} / \mathrm{L}$ concentration. We excited the molecules with a linearly polarized mode-locked Ti:sapphire laser, with a repetition rate of $76 \mathrm{MHz}$, frequency-doubled to $459 \mathrm{~nm}$ (Coherent, model Mira 900). A polarizing beamsplitter separated the parallel and perpendicular components of the fluorescence emission which were focused onto a pair of avalanche photodiodes with a full-width half-max (FWHM) response of less than 40 ps (Micro Photon Devices, Bolzano, Italy, model PDM 50ct). The parallel and perpendicular intensity histograms were collected using time-correlated single photon counting (TCSPC) electronics with 3 ps A/D resolution (Becker \& Hickl, Berlin, Germany, model SPC-830).

The measured photon counts $I_{\| \mid}$and $I_{\perp}$ collected on each of the two detectors is the fluorescence decay convolved with the instrument response functions (IRF) in each channel such that 


$$
\begin{aligned}
& I_{\|}=I R F_{\|} * i_{\|} \\
& I_{\perp}=I R F_{\perp} * i_{\perp},
\end{aligned}
$$

where $i_{\|}$is emitted light with polarization parallel to the excitation light and $i_{\perp}$ is emitted light with polarization perpendicular to the excitation light and $I R F_{\|}$and $I R F_{\perp}$ are the respective IRFs for each channel. The IRFs were measured using the water Raman line. The fluorescence decay of a freely rotating molecule has the generic form. ${ }^{18,19}$

$$
\begin{aligned}
& i_{\|}=A_{\|} \exp (-t / \tau)[1+2 \alpha \exp (-t / \theta)] \\
& i_{\perp}=A_{\perp} \exp (-t / \tau)[1-\alpha \exp (-t / \theta)]
\end{aligned},
$$

where $A_{\|}$and $A_{\perp}$ are overall amplitude terms, $\alpha$ is the fundamental anisotropy, $\tau$ is the fluorescence lifetime, and $\theta$ is the rotational correlation time.

\subsection{Rotational dynamics of EGFP encapsulated in hydrosomes}

In our preliminary analysis of EGFP, we found that the fluorescence decay fit best with two lifetime decay components, and one rotational decay component. The presence of two lifetime decay components is consistent previous studies of GFP fluorescence decay. ${ }^{20,21}$ The fluorescence decay of EGFP was fit, with a global fitting routine, to

$$
\begin{aligned}
& I_{\|}=I R F *\left\{A_{\|}\left(\exp \left(-t / \tau_{1}\right)+a \exp \left(-t / \tau_{2}\right)\right)[1+2 \alpha \exp (-t / \theta)]\right\} \\
& I_{\perp}=I R F *\left\{A_{\perp}\left(\exp \left(-t / \tau_{1}\right)+a \exp \left(-t / \tau_{2}\right)\right)[1-\alpha \exp (-t / \theta)]\right\}
\end{aligned}
$$

Figure 3 shows typical fluorescence polarization decay curves for EGFP in hydrosomes and in free solution. We made 31 fluorescence polarization decay measurements of EGFP encapsulated in hydrosomes at a concentration of $3 \mu \mathrm{mol} / \mathrm{L}$ and 10 measurements of EGFP in free solution at the same concentration. Each of these measurements was fit to equation (3), from which we extracted a rotational correlation time. The rotational correlation times we state here are an unweighted average of the fit results from each measurement, and the stated uncertainty is the standard deviation of these results. We note that the error on the rotational time for each fit is typically larger than the standard deviation of the fit results. Preliminary analysis using this technique indicates that the mean rotational time of all of the measurements of hydrosome encapsulated EGFP was $19 \mathrm{~ns} \pm 10 \mathrm{~ns}$. For EGFP in free solution, the mean rotational time was $16 \mathrm{~ns} \pm 2 \mathrm{~ns}$. These results are consistent with previous reports for EGFP and wild-type GFP where the rotational times were found to be approximately $15 \mathrm{~ns}$ to $20 \mathrm{~ns}$ in bulk water. ${ }^{20,22}$

\subsection{Rotational dynamics of 20mer DNA duplex encapsulated in hydrosomes}

Preliminary analysis indicates that the 20mer DNA duplex labeled with Alexa 488 is best fit to a function having one lifetime decay component and two rotational decay components. There are two possible explanations for the second rotational time: the tethered dye may have some degree of rotational freedom ${ }^{23}$ or it is possible we are measuring more than one rotational time for the DNA, since it is better approximated by rigid rod than a sphere ${ }^{24}$. The fluorescence decay of the labeled DNA was fit to

$$
\begin{aligned}
& I_{\|}=I R F *\left\{A_{\|} \exp (-t / \tau)\left[1+2\left(\alpha_{1} \exp \left(-t / \theta_{1}\right)+\alpha_{2} \exp \left(-t / \theta_{2}\right)\right)\right]\right\} \\
& I_{\perp}=I R F *\left\{A_{\perp}\left(\exp (-t / \tau)\left[1-\left(\left(\alpha_{1} \exp \left(-t / \theta_{1}\right)+\alpha_{2} \exp \left(-t / \theta_{2}\right)\right)\right)\right]\right\}\right.
\end{aligned} .
$$

Typical fluorescence polarization decay curves for DNA in hydrosomes and in free solution are shown in Figure 4. We made 10 fluorescence decay measurements of DNA encapsulated in hydrosomes at a concentration of $3 \mu \mathrm{mol} / \mathrm{L}$, and 2 measurements of DNA in free solution at the same concentration. Preliminary analysis indicates that the mean rotational times, $\theta_{1}$ and $\theta_{2}$, of the hydrosome encapsulated DNA are $1.4 \mathrm{~ns} \pm 0.2 \mathrm{~ns}$, and $0.24 \mathrm{~ns} \pm 0.03 \mathrm{~ns}$. The mean 
rotational times of the free DNA are $2.50 \mathrm{~ns} \pm 0.04 \mathrm{~ns}$, and $0.270 \pm 0.001 \mathrm{~ns}$. As explained in the previous section, the stated mean values are an unweighted average of the fit results from each measurement, and the uncertainties are the standard deviation spread of these results. We note that preliminary analysis indicates the rotational times in the hydrosomes are faster than in free solution, a result we do not yet understand.

\subsection{Conclusions from anisotropy measurements}

If the molecules were sticking or aggregating at the boundary interface, we would observe a much longer rotational correlation time when the molecules are encapsulated in the hydrosome compared to bulk solution. That is clearly not the case for both EGFP and DNA. From measurement of EGFP, since the rotational times inside the hydrosome and in bulk solution are equal within error, we conclude that the protein rotates freely in hydrosomes. For DNA, the rotational dynamics are clearly different inside and outside the hydrosome, but there is still no obvious sticking or aggregation at the boundary as the molecules appear to rotate faster than in bulk.

\section{USING HYDROSOMES FOR NANOCHEMISTRY}

The most exciting advantage of using hydrosomes over other femtoliter containers such as liposomes is that hydrosomes spontaneously fuse when they are brought into contact, as shown in Figure 5. Figure 5 also shows a demonstration of contents within hydrosomes mixing upon fusion. The hydrosomes in this particular experiment were produced by the ultrasonication method. In the figure, one of the hydrosomes contains $1 \mu \mathrm{mol} / \mathrm{L}$ of single-stranded DNA (ssDNA) labeled with a Cy3 fluorophore (the FRET donor) and the other hydrosome contains $1 \mu \mathrm{mol} / \mathrm{L}$ of the complementary strand labeled with a Cy5 fluorophore (the FRET acceptor). The DNA used in this experiment was purchased from IDT. The visible probe laser we use to excite the Cy3 fluorophore is at $532 \mathrm{~nm}$ (CrystalLaser GLC-532-L). The hydrosome containing ssDNA labeled with Cy5, which is only very weakly excited by our probe laser, is held in the trap, while the hydrosome containing ssDNA labeled with Cy3 is brought into contact with the first hydrosome via a second optical trap. As the Cy3 labeled ssDNA enters the observation volume, it is excited by the probe laser, and the signal in the donor channel starts to increase. As the ssDNA hybridizes and becomes a duplex, a FRET signal is observed. FRET will occur only as the DNA hybridizes, when the two fluorophores are sufficiently close that the donor transfers its energy to the acceptor. The sharp rise in acceptor signal is accompanied by a sharp drop in donor signal, which is the signature of FRET. We have hence shown that a simple reaction, such as DNA hybridization, can be induced by hydrosome mixing.

Mixing hydrosomes permits the study of single transiently interacting molecular complexes. One can observe a reaction between two individual molecules by bringing together two hydrosomes each containing a distinct species. We can also use hydrosome fusion to quickly change the buffer environment for a molecule under study. The ability to fuse one hydrosome to another permits direct observation of transient non-equilibrium states and reaction pathways.

\section{CONCLUSIONS}

We have shown that we can produce subfemtoliter droplets, hydrosomes, on demand, and that molecules encapsulated within hydrosomes are not aggregating or sticking at the hydrophobic boundary. Hydrosomes are used to encapsulate molecules for single molecule or other fluorescence spectroscopy studies and offer a promising method for undertaking nanochemistry experiments. 

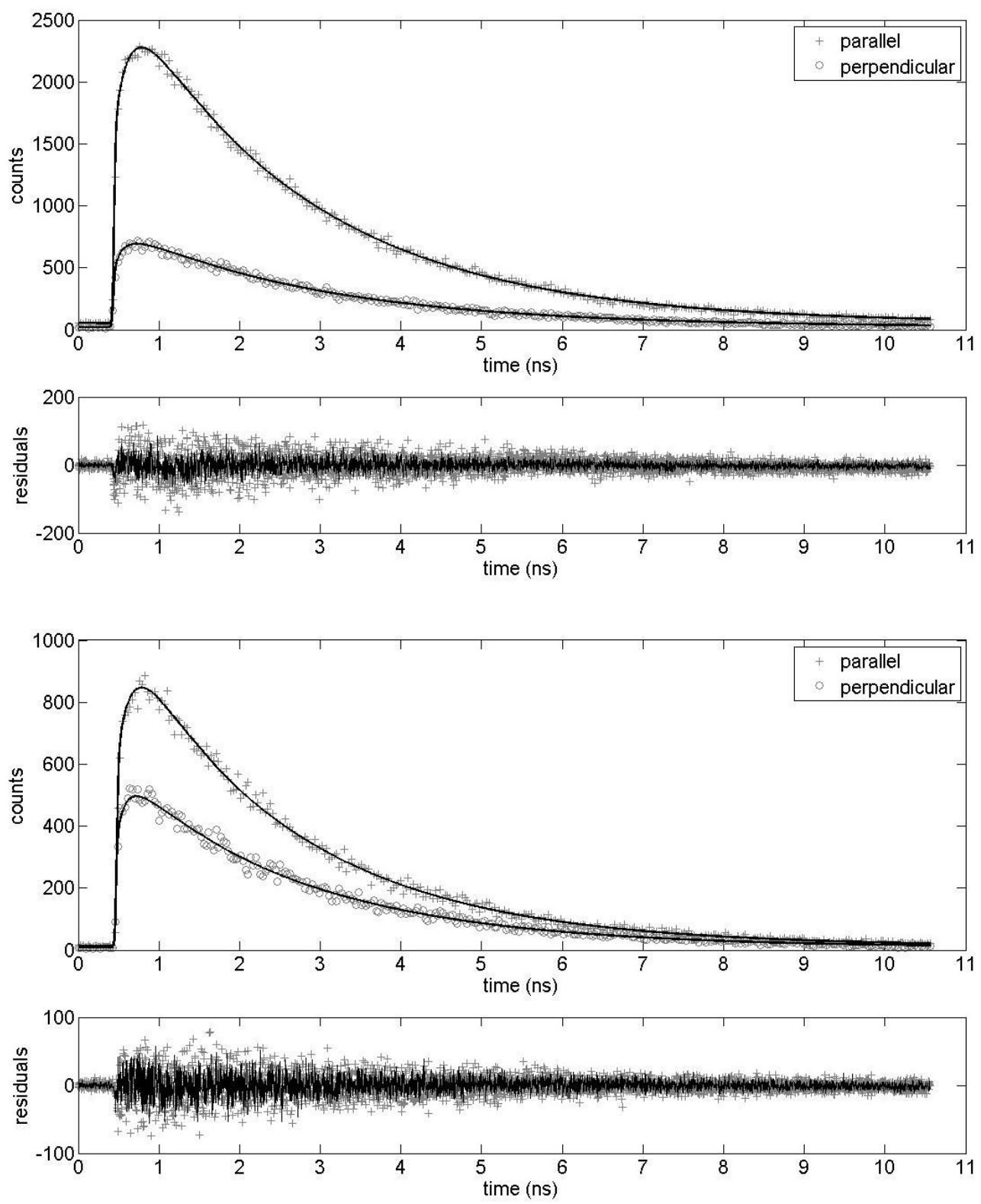

Figure 3: (Top) Fluorescence polarization decay of $3 \mu \mathrm{mol} / \mathrm{L}$ EGFP in bulk solution. (Bottom) Fluorescence polarization decay of $3 \mu \mathrm{mol} / \mathrm{L}$ EGFP encapsulated in a hydrosome. The solid lines are the fit, and the points are the data. For visual clarity, the plots show only one out of every ten data points. The corresponding residuals are shown below each fit. In the residuals, the points correspond to the parallel fit, and the solid line corresponds to the perpendicular fit. 

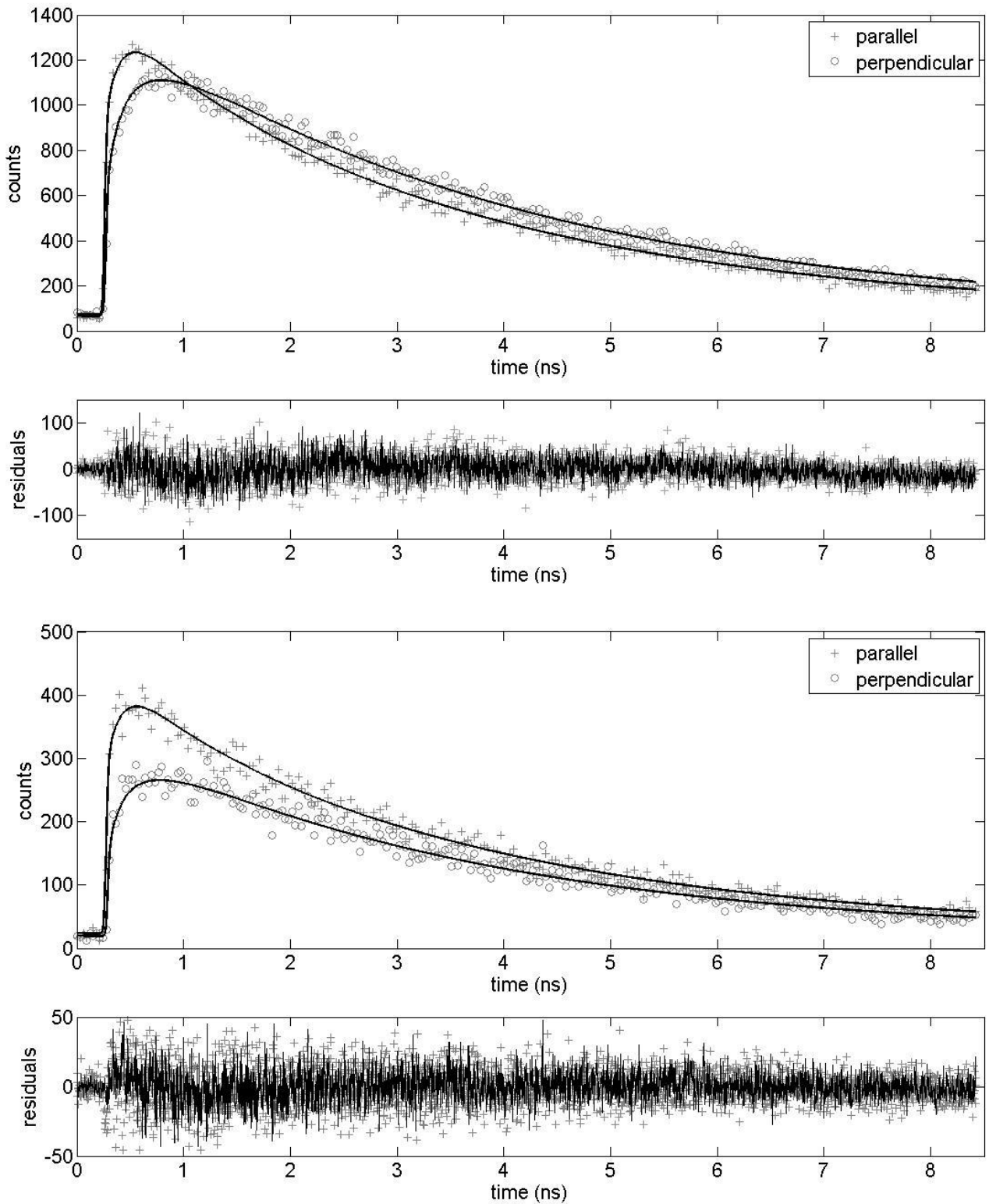

Figure 4: (Top) Fluorescence polarization decay of $3 \mu \mathrm{mol} / \mathrm{L} 20 \mathrm{mer}$ DNA duplex in bulk solution. (Bottom) Fluorescence polarization decay of $3 \mu \mathrm{mol} / \mathrm{L} 20 \mathrm{mer}$ DNA duplex encapsulated in a hydrosome. The DNA was labeled with the Alexa 488 fluorophore. The solid lines are the fit, and the points are the data. For visual clarity, the plots show only one out of every ten data points. The corresponding residuals are shown below each fit. In the residuals, the points correspond to the parallel fit, and the solid line corresponds to the perpendicular fit. 


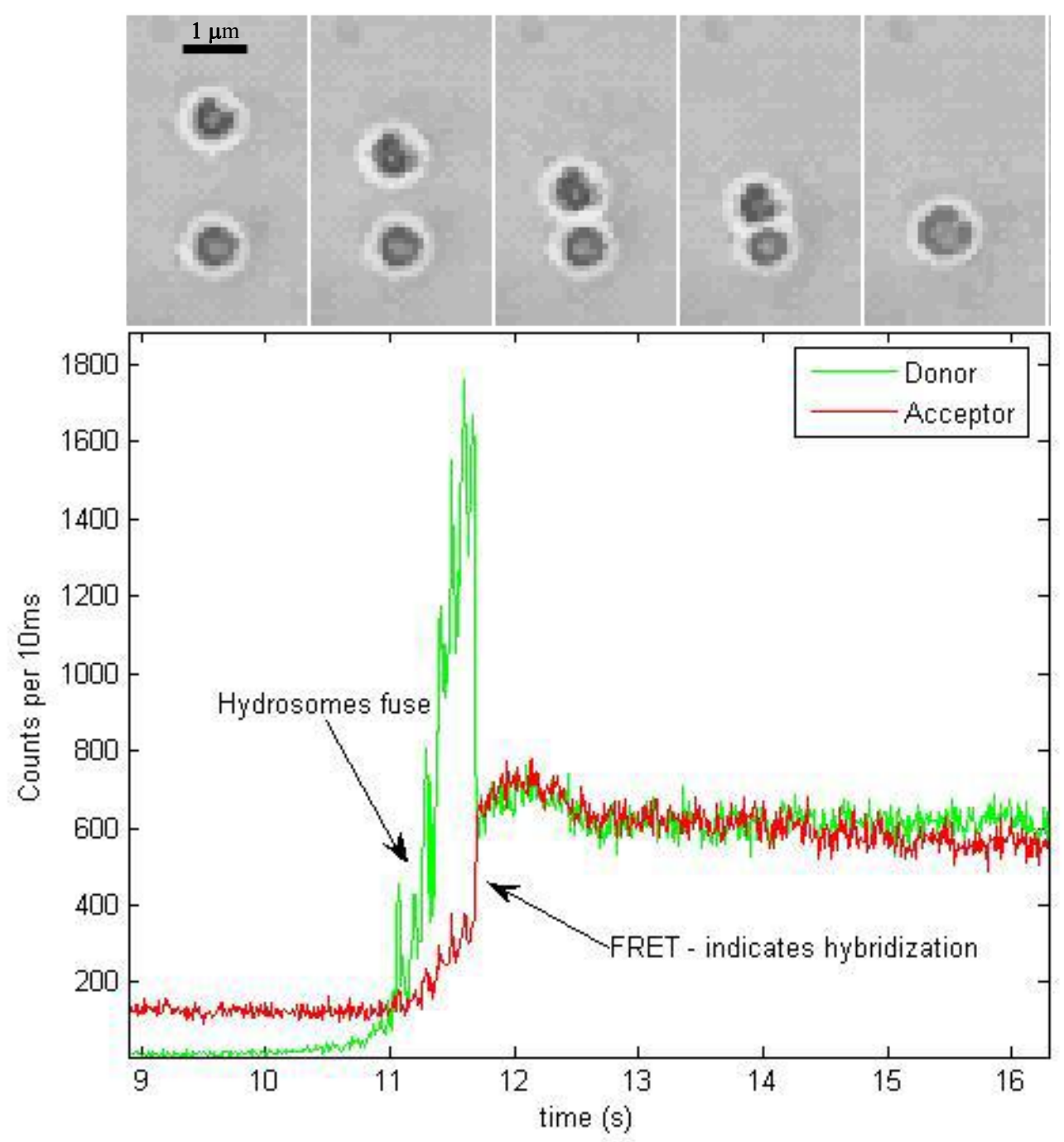

Figure 5: (Top) Two hydrosomes are brought into contact by means of manipulation with optical tweezers. The optical traps are made using an infrared laser at $1064 \mathrm{~nm}$ (IPG photonics YLD-51064). This figure demonstrates that two hydrosomes fuse when they are brought into contact with one another. (Bottom) Here we demonstrate DNA hybridization that occurs when two hydrosomes are fused. One hydrosome contains $1 \mu \mathrm{mol} / \mathrm{L}$ of ssDNA labeled with a Cy5 fluorophore (the acceptor), and the other hydrosome contains $1 \mu \mathrm{mol} / \mathrm{L}$ of the complementary ssDNA labeled with a Cy3 fluorophore (the donor). We use a $532 \mathrm{~nm}$ laser (CrystaLaser GLC-532-L) to probe the molecules. The hydrosome containing the acceptor is held in the observation volume while the hydrosome containing the donor is brought into contact. As the donor molecules enter the observation volume, they are excited by the probe laser, and the signal in the donor channel begins to increase. The acceptor signal also increases just before fusion due a slight spectral overlap of donor and acceptor emission wavelengths. The subsequent sharp rise in acceptor signal, accompanied by a sharp drop in donor signal, is the signature of FRET. In this system, FRET will occur only when the DNA hybridizes, when the two fluorophores are sufficiently close that the donor transfers its energy to the acceptor. 


\section{ACKNOWLEDGMENTS}

We acknowledge funding from the Office of Naval Research (ONR), the National Research Council (NRC), and the NIST Physics Laboratory. The FC-70 used in this work was a generous gift from 3M. We thank Joseph Reiner, Kimberly Briggman, John Denker, and Jeeseong Hwang for helpful discussions. We also thank Joseph Reiner for the schematic diagram of the apparatus shown in Figure 1. Certain commercial equipment, instruments, or materials are identified in this paper to foster understanding. Such identification does not imply recommendation or endorsement by the National Institute of Standards and Technology, nor does it imply that the materials or equipment identified are necessarily the best available for the purpose.

\footnotetext{
${ }^{1}$ Weiss, S. "Measuring conformational dynamics of biomolecules by single molecule fluorescence spectroscopy." Nat. Struct. Biol. 7, 724 (2000).

2 Eigen, M.; Rigler, R. "Sorting Single Molecules: Application to Diagnostics and Evolutionary Biotechnology." Proc. Nat. Acad. Sci. U.S.A. 91, 5740 (1994)

${ }^{3}$ Ha, T.; Zhuang, X.; Kim, H. D.; Orr, J.W.; Williamson, J.R.; and Chu, S. "Ligand-induced conformational changes observed in single RNA molecules.” Proc. Natl. Acad. Sci. U.S.A. 96, 9077 (1999).

${ }^{4}$ Wennmalm, S.; Edman, L.; R. Rigler, R. "Conformational fluctuations in single DNA molecules.” Proc. Natl. Acad. Sci. U.S.A. 94, 10641 (1997).
}

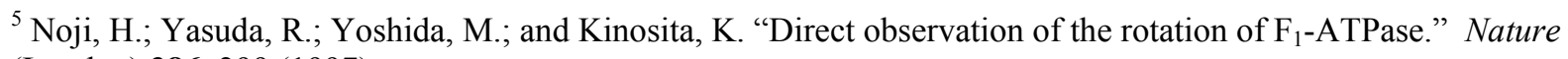
(London) 386, 299 (1997).

${ }^{6}$ Jia,Y.; Talaga, D. S.; Lau, W. L.; Lu, H. S. M.; DeGrado, W. F.; and Hochstrasser, R. M. "Folding dynamics of single GCN-4 peptides by fluorescence resonant energy transfer confocal microscopy." Chem. Phys. 247, 69 (1999).

${ }^{7}$ Talaga, D. S.; Lau, W. L.; Roder, H.; Tang, J. Y.; Jia, Y.; DeGrado, W. F.; and Hochstrasser, R. M. "Dynamics and folding of single two-stranded coiled-coil peptides studied by fluorescent energy transfer confocal microscopy." Proc. Natl. Acad. Sci. U.S.A. 97, 13021 (2000).

${ }^{8}$ Bopp, M. A.; Jia, Y. W.; Li, L. Q.; Cogdell, R. J.; and Hochstrasser, R. M. "Fluorescence and photobleaching dynamics of single light-harvesting complexes.” Proc. Natl. Acad. Sci. U.S.A. 94, 10630 (1997).

${ }^{9}$ Wazawa, T.; Ishii, Y.; Funatsu, T.; and Yanagida, T. "Spectral Fluctuation of a Single Fluorophore Conjugated to a Protein Molecule.” Biophys. J. 78, 1561 (2000).

${ }^{10}$ Dickson, R. M.; Cubitt, A. B.; Tsien, R. Y.; and Moerner, W. E. "On/off blinking and switching behaviour of single molecules of green fluorescent protein." Nature (London) 388, 355 (1997).

${ }^{11}$ Lu, H. P.; Xu, L. Y.; and Xie, X. S. “Single-Molecule Enzymatic Dynamics.” Science 282, 1877 (1998) 
${ }^{12}$ Boukobza, E.; Sonnenfeld, A.; and Haran, G. "Immobilization in Surface-Tethered Lipid Vesicles as a New Tool for Single Biomolecule Spectroscopy.” J. Phys. Chem. B 105, 12165 (2001)

${ }^{13}$ Rhoades, E.; Gussakovsky, E.; and Haran, G. "Watching proteins fold one molecule at a time." Proc. Natl. Acad. Sci. U.S.A. 100, 3197 (2003)

${ }^{14}$ Okumus, B.; Wilson, T. J.; Lilley, D. M. J.; and Ha, T. "Vesicle Encapsulation Studies Reveal that Single Molecule Ribozyme Heterogeneities Are Intrinsic.” Biophys. J. 87, 2798 (2004)

${ }^{15}$ Reiner, J.E.; Crawford, A.M.; Kishore, R.B.; Goldner, L.S.; Gilson, M.K.; Helmerson, K. “Optically Trapped Aqueous Droplets for Single Molecule Studies.” Appl. Phys. Lett. 89, 013904 (2006)

${ }^{16}$ Zhang, W.; Hou, L.; Mu,L.; Zhu, L.; "Femtoliter micro-injector using digital microfluidic control." Proceedings of SPIE 5345 Microfluidics, BioMEMS, and Medical Microsystems II, 220 (2004)

${ }^{17}$ Lee, C.H.; Lal, A. "Single Microdroplet Ejection using an Ultrasonic Longitudinal Mode with a PZT/Tapered Glass Capillary." IEEE Transactions on Ultrasonics Ferroelectrics and Frequency Control 51, 1514 (2004)

${ }^{18}$ Lakowicz, Joseph R. Principles of Fluorescence Spectroscopy. Chapter 10-12, Springer: New York 2006

${ }^{19} \mathrm{Hu}$, D.; Lu, H.P. "Single-Molecule Nanosecond Anisotropy Dynamics of Tethered Protein Motions." J. Phys. Chem. $B$ 107, $618(2003)$

${ }^{20}$ Volkmer, A.; Subramaniam, V.; Birch, D. J. S.; Jovin, T. M. "One- and Two-Photon Excited Fluorescence Lifetimes and Anisotropy Decays of Green Fluorescent Proteins.” Biophys. J. 78, 1589 (2000).

${ }^{21}$ Striker, G.; Subramaniam, V.; Seidel, C. A. M.; Volkmer, A. "Photochromicity and Fluorescence Lifetimes of Green Fluorescent Protein" J. Phys. Chem B 103, 8612 (1999).

${ }^{22}$ Swaminathan, R; Hoang, C. P.; Verkman, A. S. Biophys. J. "Photobleaching recovery and anisotropy decay of green fluorescent protein GFP-S65T in solution and cells: cytoplasmic viscosity probed by green fluorescent protein translational and rotational diffusion." 72, 1900 (1997).

${ }^{23}$ Kumke, M.U.; Li, G.; McGown L.B.; Walker G.T.; Linn, C.P. "Hybridization of Fluorescein-Labeled DNA Oligomers Detected by Fluorescence Anisotropy with Protein Binding Enhancement.” Anal. Chem. 67, 3945 (1995)

${ }^{24}$ Hogan, M.; Wang, J.; Austin, R.H.; Monitto, C.L.; Hershkowitz, S. "Molecular motion of DNA as measured by triplet anisotropy decay" Proc. Natl. Acad. Sci. USA 79, 3518 (1982) 